https://helda.helsinki.fi

Dexamethasone in head and neck cancer patients with microvascular reconstruction : No benefit, more complications

Kainulainen, S.

2017-02

Kainulainen , S , Tornwall , J , Koivusalo , A M , Suominen , A L \& Lassus , P 2017 , '

Dexamethasone in head and neck cancer patients with microvascular reconstruction: No

benefit, more complications ' , Oral Oncology , vol. 65 , pp. 45-50 . https://doi.org/10.1016/j.oraloncology.2016.12.00

http://hdl.handle.net/10138/233302

https://doi.org/10.1016/j.oraloncology.2016.12.008

publishedVersion

Downloaded from Helda, University of Helsinki institutional repository.

This is an electronic reprint of the original article.

This reprint may differ from the original in pagination and typographic detail.

Please cite the original version. 


\title{
Dexamethasone in head and neck cancer patients with microvascular reconstruction: No benefit, more complications
}

\author{
S. Kainulainen ${ }^{\text {a,* }}$, J. Törnwall ${ }^{\text {a }}$, A.M. Koivusalo ${ }^{c}$, A.L. Suominen ${ }^{\text {d,e }}$, P. Lassus ${ }^{b}$ \\ a Department of Oral and Maxillofacial Surgery, Helsinki University Hospital, University of Helsinki, Helsinki, Finland \\ ${ }^{\mathrm{b}}$ Department of Plastic Surgery, Helsinki University Hospital, University of Helsinki, Helsinki, Finland \\ ${ }^{\mathrm{c}}$ Department of Anesthesia and Intensive Care Unit, Helsinki University Central Hospital, Helsinki, Finland \\ ${ }^{\mathrm{d}}$ Institute of Dentistry, University of Eastern Finland, Kuopio, Finland \\ e Department of Oral and Maxillofacial Surgery, Kuopio University Hospital, Kuopio, Finland
}

\section{A R T I C L E I N F O}

\section{Article history:}

Received 18 October 2016

Received in revised form 29 November 2016

Accepted 10 December 2016

Available online 27 December 2016

\section{Keywords:}

Glucocorticoid

Dexamethasone

Head and neck cancer

Microvascular reconstruction

Complications

\begin{abstract}
A B S T R A C T
Objectives: Glucocorticoids are widely used in association with major surgery of the head and neck to improve postoperative rehabilitation, shorten intensive care unit and hospital stay, and reduce neck swelling. This study aimed to clarify whether peri- and postoperative use of dexamethasone in reconstructive head and neck cancer surgery is associated with any advantages or disadvantages.

Materials and methods: This prospective double-blind randomized controlled trial comprised 93 patients. A total dose of $60 \mathrm{mg}$ of dexamethasone was administered to 51 patients over three days peri- and postoperatively. The remaining 42 patients served as controls. The main primary outcome variables were neck swelling, length of intensive care unit and hospital stay, duration of intubation or tracheostomy, and delay to start of possible radiotherapy. Complications were also recorded.

Results: No statistical differences emerged between the two groups in any of the main primary outcome variables. However, there were more major complications, especially infections, needing secondary surgery within three weeks of the operation in patients receiving dexamethasone than in control patients (27\% vs. $7 \%, \mathrm{p}=0.012$ ).

Conclusions: The use of dexamethasone in oral cancer patients with microvascular reconstruction did not provide a benefit. More major complications, especially infections, occurred in patients receiving dexamethasone. Our data thus do not support the use of peri- and postoperative dexamethasone in oropharyngeal cancer patients undergoing microvascular reconstruction.
\end{abstract}

(C) 2016 Elsevier Ltd. All rights reserved.

\section{Introduction}

Treatment of oropharyngeal carcinoma causes major morbidity. Treatment modalities include surgery, radiation, and chemotherapy. In surgery, microvascular tissue reconstruction has become the definitive method to cover large defects after tumor resection. Reconstruction improves the healing and is essential to restore oral function and esthetics, thereby improving the quality of life after an extensive surgical procedure [1-8]. Oropharyngeal tumor surgery is associated with many postoperative problems like respiratory problems, major swelling, prolonged length of tracheostomy, and lengthened intensive care unit and hospital stay. A major goal in treatment is to achieve primary healing without delaying possible adjuvant radiotherapy.

* Corresponding author at: Department of Oral and Maxillofacial Surgery, Helsinki University Hospital, P.O. Box 220, FI-00029 HUS Helsinki, Finland.

E-mail address: satu.kainulainen@hus.fi (S. Kainulainen).
Glucocorticoids (GCs) are widely used in association with head and neck surgery to reduce these undesirable problems due their anti-inflammatory effects [9-11]. GCs improve postoperative rehabilitation, shorten intensive care unit and hospital stay, and reduce neck swelling. However, adverse effects may follow GC use, particularly when high doses are used. Short-term GC use has been reported to increase the risk for avascular necrosis of the femoral head, steroid-induced psychosis, peptic ulcers, and gastrointestinal bleeding [12-21]. Another considerable disadvantage of steroids is impaired wound healing, which may increase postoperative infections and complications [22-24]. Despite the well-documented efficacy of systemic dexamethasone usage in surgery, no data exist regarding effects and safety of dexamethasone in oropharyngeal cancer reconstructive surgery.

We performed a prospective randomized double-blind control study to examine whether the peri- and postoperative use of dex- 
amethasone in reconstructive head and neck cancer surgery is associated with advantages or disadvantages.

\section{Materials and methods}

A prospective double-blind randomized controlled trial was conducted between December 2008 and February 2013 at the Department of Oral and Maxillofacial Surgery and the Department of Plastic Surgery, Helsinki University Hospital, Finland. The study protocol was approved by the Research Ethics Committee of Helsinki University Hospital. Written informed consent was obtained from all patients before surgery.

Consecutive patients with oropharyngeal cancer who had a microvascular reconstruction were included in the study. Exclusion criteria were history of liver or kidney dysfunction, glaucoma, peptic ulcer, psychosis from use of steroids, allergy to any constituent of the dexamethasone preparation used, and absence of written informed consent. We collected consecutive 110 patients, 55 to each group. Ninety-seven patients met the inclusion criteria. Four of these patients were subsequently excluded, three because of intraoperative cancellation of free flap reconstruction and one because he was administered additional dexamethasone. Therefore, 93 patients were included in the study, 73 from the Department of Maxillofacial Surgery and 20 from the Department of Plastic Surgery. Of the 93 patients, 51 had received dexamethasone (DEX-group) and 42 had not received dexamethasone and were the control group (NON-DEX). The discrepancy in the size of two groups is explained by the effect of luck since patients were chosen to either group by random selection.

Patients were randomly allocated into two groups. The patients in the study group received dexamethasone (Oradexon ${ }^{\circledR}$ ) $10 \mathrm{mg}$ intravenously (i.v.) every $8 \mathrm{~h}$ on the first day, every $12 \mathrm{~h}$ on the second day, and one dose on the third day, receiving a total dose of $60 \mathrm{mg}$ (DEX). The patients in the control group received no dexamethasone (NON-DEX). The randomization was done by a nurse not participating in the study. The information about whether a patient would receive dexamethasone was given in a sealed envelope to the anesthesiologist in charge of the anesthesia of the surgery. The same anesthesiologist administered all doses to the patient if allocated during the operation and in the ICU postoperatively. Surgeons were unaware of the group to which patients were assigned.

Preoperative and predictive data in DEX and NON-DEX groups are given in Table 1 . The majority (92\%) of tumors were squamous cell carcinomas. The oral tongue and mandible were the most common sites of malignancy, each affected in $29 \%$ of cases. These were followed by the maxilla (16\%), floor of the mouth (12\%), buccal mucosa (10\%), tonsilla (3\%), palate (1\%), and larynx - hypopharynx (1\%). There were 83 fasciocutaneous and 10 osseofasciocutaneous reconstructions. The radial forearm (RFF) was the most frequently performed flap (31 in DEX, 20 in NON-DEX), followed by the anterolateral thigh (ALT) perforator flap (15 in DEX, 18 in NONDEX). The other flap types included the deep circumflex iliac artery (DCIA) bone flap, fibula free flap, latissimus dorsi (LD) muscle flap, and scapula or parascapular flaps. The surgical data are given in Table 2 and TNM classifications in Table 5. We classified surgical complications according to Dindo et al. so that all major complications were included to complication group IIIb or worse and received secondary surgery within three weeks $[25,26]$.

All patients received standard, balanced anesthesia. Patients received cefuroxime $1.5 \mathrm{~g} \times 3$ i.v. and metronidazole $500 \mathrm{mg}$ $1 \times 3$ i.v. over an average of 7 days, starting from induction of general anesthesia. Patients with allergies were given clindamycin $300 \mathrm{mg} \times 4$ i.v. In the postoperative period, patients were given paracetamol $1 \mathrm{~g} \times 3$ i.v. No non-steroidal anti-inflammatory drugs were used. Postoperatively, oxycodone $0.2-0.4 \mathrm{mg} / 10 \mathrm{~kg}$ i.v. was administered if the patient scored more than 4 on a Visual Analog Scale (VAS) or when requested by the patient. Postoperative nausea was treated with ondansetron as needed. One physician (SK) collected and sorted the data from the follow-up forms and hospital database.

\section{Statistical analysis}

Significance of associations between groups and categorical variables was evaluated by Chi-square tests. Differences in mean values between groups and continuous variables were evaluated by Student's $t$-tests for normally distributed variables and by Wilcoxon two-sample test for variables with skewed distributions. To indicate overall recovery from the surgery, a score was formed that included the following 12 continuous variables: change in neck circumference, start of using Heat and Moisture Exchanger (HME), time of decannulation of tracheostomy/extubation, neck drainage removal time, start of communication, sitting, standing, walking, drinking fluids, transferring to the hospital ward and home, and change in patient's weight during hospital stay. Each variable was at first categorized according to median value $(-1$ if $\leqslant$ median and +1 if $>$ median). A sum score of these was calculated and further dichotomized according to median value (median $\geqslant 7 ; 1$ indicating shorter/better recovery, median $\geqslant 7 ; 0$ indicating longer/ poorer recovery, median $<7$ ) to serve as an outcome in logistic regression analysis. Explanatory variables included group (NONDEX or DEX), age as continuous, sex, Body Mass Index (BMI), history of alcohol use (major, moderate, or none), length of surgery, American Society of Anesthesiologists (ASA) score, and major complications. We did a power analysis to evaluate the statistical reliability of the present study.

\section{Results}

No statistical differences existed between the DEX and NONDEX groups regarding preoperative demographic data or preoperative treatments given to patients, except that there were more heavy alcohol users in the NON-DEX group (DEX $n=8(16 \%)$, NON-DEX $n=13(31 \%), p=0.113)$. Localizations of the tumors, TNM classifications, and surgical defects were similar between the groups. The flap types used as well as the neck dissection types and operation times were also similar between DEX and NON-DEX groups. In the NON-DEX group, more patients were tracheostomized at the beginning the operation than in the DEX group ( $60 \%$ vs. $33 \%, p=0.034)$. Postoperative adjuvant treatments were similar in both groups. There were more diabetics in the DEX group, however this difference was not statistically significant (Tables 1 and 2).

The main primary outcome variables were neck swelling, length of intensive care unit stay and hospital stay, duration of intubation or tracheostomy, and delay to start of radiotherapy. No statistical differences existed between the two groups in any of these variables. Patients' neck swelling was measured daily from the highest point of the neck for seven days postoperatively, and the highest increase in neck circumference $(\mathrm{cm})$ relative to the preoperative circumference was used in analysis. Length of tracheostomy was three days shorter (23\%) in the DEX group, but the difference was not significant. Four primarily intubated patients in the DEX group and one patient in the NON-DEX group were tracheostomized postoperatively due to prolonged need for mechanically assisted ventilation. Therefore, the total number of tracheostomies was $21 / 51$ patients (41\%) in the DEX group and $26 / 42$ patients (62\%) in the NON-DEX group (Table 3 ).

The DEX group had more major complications during the postoperative period ( $27 \%$ in DEX vs. $7 \%$ in NON-DEX, $p=0.012$ ). The 
Table 1

Patient data.

\begin{tabular}{|c|c|c|c|c|}
\hline & All $(n=93)$ & $\operatorname{DEX}(\mathrm{n}=51)$ & NON-DEX $(n=42)$ & $\mathrm{P}$ \\
\hline \multicolumn{5}{|l|}{ Demographic characteristics and comorbidities } \\
\hline Age (years) & $65(34-93)$ & $65(39-93)$ & $65(34-88)$ & 0.798 \\
\hline Male/Female & $59 / 34$ & $32 / 19$ & $27 / 15$ & 0.878 \\
\hline BMI & $24.9(15.8-42.7)$ & $25.5(15.8-42.70)$ & $24.5(17.0-32.6)$ & 0.331 \\
\hline $\operatorname{ASA}(1 / 2 / 3 / 4)$ & $6 / 23 / 48 / 13$ & $3 / 10 / 27 / 11$ & $3 / 13 / 21 / 4$ & 0.339 \\
\hline History of alcohol use (major/moderate/no) & $21 / 45 / 27$ & $8 / 23 / 20$ & $13 / 22 / 7$ & 0.038 \\
\hline History of smoking (yes/no) & $37 / 56$ & $19 / 32$ & $18 / 24$ & 0.583 \\
\hline $\mathrm{CCI}(0-1 / 2-4 / 5-9)$ & $49 / 29 / 15$ & $24 / 19 / 8$ & $25 / 10 / 7$ & 0.363 \\
\hline Diabetes & $15 / 93(18 \%)$ & $11 / 51(22 \%)$ & $4 / 42(10 \%)$ & 0.116 \\
\hline \multicolumn{5}{|l|}{ Preoperative characteristics } \\
\hline Previous radiotherapy & $9 / 93(10 \%)$ & $5(10 \%)$ & $4(10 \%)$ & 0.939 \\
\hline Previous chemotherapy & $3 / 93(3 \%)$ & $1 / 51(2 \%)$ & $2 / 42(5 \%)$ & 0.447 \\
\hline Previous operation in same area & $14 / 91(15 \%)$ & $10 / 49(20 \%)$ & $4 / 42(10 \%)$ & 0.151 \\
\hline \multicolumn{5}{|l|}{ Perioperative data } \\
\hline Tracheostomy / intubation & $47(51 \%) / 46(49 \%)$ & $21(41 \%) / 30(59 \%)$ & $26(62 \%) / 16(34 \%)$ & 0.047 \\
\hline \multicolumn{5}{|l|}{ Postoperative data } \\
\hline Radiation therapy postoperatively & $45(50 \%)$ & $20(43 \%)$ & $25(58 \%)$ & 0.211 \\
\hline Chemotherapy postoperatively & $20(23 \%)$ & $8(17 \%)$ & $12(29 \%)$ & 0.326 \\
\hline
\end{tabular}

DEX: dexamethasone group.

NON-DEX: non-dexamethasone group.

BMI: Body Mass Index.

ASA: American Society of Anesthesiologists.

CCI: Charlson Comorbidity Index.

Alcohol use was defined as moderate if drinking was weekly or less and major if it occurred daily.

Patients were defined as smokers if they smoked before surgery.

Data given as median and range.

Table 2

Surgical data.

\begin{tabular}{|c|c|c|c|c|}
\hline & All $(n=93)$ & $\operatorname{DEX}(\mathrm{n}=51)$ & NON-DEX $(n=42)$ & $\mathrm{P}$ \\
\hline Reconstruction type: Soft tissue/bone & $83 / 10$ & $46 / 5$ & $37 / 5$ & 0.745 \\
\hline \multicolumn{5}{|l|}{ Primary lesion } \\
\hline Tongue & 27 & 13 & 14 & \\
\hline Floor of mouth & 11 & 8 & 3 & \\
\hline Mandible & 26 & 14 & 12 & \\
\hline Maxilla & 15 & 9 & 6 & \\
\hline Buccal mucosa & 9 & 5 & 4 & \\
\hline Tonsilla & 3 & 1 & 2 & \\
\hline Palate & 1 & 1 & 0 & \\
\hline Larynx - hypofarynx & 1 & 0 & 1 & \\
\hline Flap type & & & & 0.360 \\
\hline Forearm flap & 51 & 31 & 20 & \\
\hline ALT & 33 & 15 & 18 & \\
\hline DCIA & 4 & 2 & 2 & \\
\hline Fibula & 1 & 1 & 0 & \\
\hline LD & 1 & 0 & 1 & \\
\hline Scapula + LD & 2 & 0 & 2 & \\
\hline Scapula + parascapula & 1 & 1 & 0 & \\
\hline Neck dissection & & & & 0.207 \\
\hline Unilateral & 78 & 45 & 33 & \\
\hline Bilateral & 15 & 6 & 9 & \\
\hline Neck dissection levels & & & & 0.201 \\
\hline Sentinel & 10 & 7 & 3 & \\
\hline L1-3 & 28 & 18 & 10 & \\
\hline $\mathrm{L} 1-4 / 5$ or radical & 55 & 26 & 29 & \\
\hline Operation time (min) & $340(87-975)$ & $340(138-975)$ & 359 (208-719) & 0.373 \\
\hline
\end{tabular}

DEX: dexamethasone group.

NON-DEX: non-dexamethasone group.

ALT: Anterolateral Thigh Perforator flap.

LD: latissimus dorsi muscle.

DCIA: The deep circumflex iliac artery bone flap.

NON-DEX group had no fistulas, wound necrosis, or surgical infections. Two patients in the DEX group and one patient in the NONDEX group had more than one major complication. Minor complications included those that only required local treatment bed-side or drug treatment and did not need surgical interventions in the operation room. Seven recorded minor complications occurred, four in the DEX group (10\%) and three in the NON-DEX group (12\%). The difference between the groups was not significant (Table 4).

Seventeen cases (18\%) developed infections within 14 days of the operation. These were all treated with a change in antibiotics and then categorized into the infection group. Infections included 
Table 3

Primary outcome measures.

\begin{tabular}{|c|c|c|c|c|}
\hline & All $(n=93)$ & $\operatorname{DEX}(n=51)$ & NON-DEX $(n=42)$ & $\mathrm{P}$ \\
\hline Neck swelling $(\mathrm{cm})(\mathrm{n}=77)^{\mathrm{a}}$ & $5.5(0-13)$ & $5.0(0-12.5)$ & $6.0(1.5-13)$ & 0.196 \\
\hline Length of ICU stay (days) $(n=93)$ & $3(1-12)$ & $3(1-12)$ & $3(1-8)$ & 0.965 \\
\hline Length of hospital stay (days) $(n=91)$ & $13(5-49)$ & $12(5-35)$ & $13(6-49)$ & 0.594 \\
\hline Duration of tracheostomy (days) $(n=46)$ & $8(2-43)$ & $6(2-18)$ & $9(2-42)$ & 0.251 \\
\hline Duration of intubation (days) $(n=47)$ & $1(0-6)$ & $1(1-6)$ & $1(0-5)$ & 0.064 \\
\hline Start of radiation therapy postoperatively (days) $(n=33)$ & $43(30-99)$ & 47 (34-99) & $43(30-74)$ & 0.110 \\
\hline
\end{tabular}

DEX: dexamethasone group.

NON-DEX: non-dexamethasone group.

Data given as median and range.

a Highest increase $(\mathrm{cm})$ in neck circumference during the seven postoperative days.

Table 4

Complications.

\begin{tabular}{|c|c|c|c|c|}
\hline & $\operatorname{ALL}(n=93)$ & $\operatorname{DEX}(\mathrm{n}=51)$ & NON-DEX $(\mathrm{n}=42)$ & $\mathrm{P}$ \\
\hline Number of major complications (patients) & $17 / 93(18 \%)$ & $14 / 51(27 \%)$ & $3 / 42(7 \%)$ & 0.012 \\
\hline Number of major complications & 20 & 16 & 4 & \\
\hline Venous thrombosis & $6(6 \%)$ & $4(8 \%)$ & $2(5 \%)$ & \\
\hline Flap loss & $3(3 \%)$ & $2(4 \%)$ & $1(2 \%)$ & \\
\hline Wound necrosis, fistula, infection & $4(4 \%)$ & $4(8 \%)$ & 0 & \\
\hline Postoperative bleeding & $1(1 \%)$ & $1(2 \%)$ & 0 & \\
\hline Later tracheostomy & $5(4 \%)$ & $4(8 \%)$ & $1(2 \%)$ & \\
\hline Pneumothorax & $1(1 \%)$ & $1(2 \%)$ & 0 & \\
\hline Number of minor complications (patients) & $7 / 93(11 \%)$ & $4 / 51(10 \%)$ & $3 / 42(12 \%)$ & 0.899 \\
\hline Fluid collection/seroma & $2(2 \%)$ & $1(2 \%)$ & $1(2 \%)$ & \\
\hline Local wound infection (neck or face) & $1(1 \%)$ & $1(2 \%)$ & 0 & \\
\hline Hematoma/bleeding & $3(3 \%)$ & $1(2 \%)$ & $2(5 \%)$ & \\
\hline Fistula & $1(1 \%)$ & $1(2 \%)$ & 0 & \\
\hline Infection postoperatively & $17 / 94(18 \%)$ & $10 / 51(20 \%)$ & $7 / 42(17 \%)$ & 0.715 \\
\hline
\end{tabular}

DEX: dexamethasone group.

NON-DEX: non-dexamethasone group.

Data given as median and range.

Table 5

TNM classification.

\begin{tabular}{|c|c|c|c|c|c|c|c|}
\hline & \multicolumn{2}{|l|}{ NO } & \multicolumn{2}{|l|}{ N1 } & \multicolumn{2}{|c|}{$\mathrm{N} 2 \mathrm{~A}-\mathrm{C}$} & \multirow[t]{2}{*}{ TOTAL } \\
\hline & DEX & NON-DEX & DEX & NON-DEX & DEX & NON-DEX & \\
\hline $\mathrm{T} 1-\mathrm{T} 2$ & 22 & 12 & 2 & 6 & 3 & 6 & 51 \\
\hline T3 - T4A-B & 12 & 10 & 2 & 1 & 9 & 6 & 40 \\
\hline TOTAL & 34 & 22 & 4 & 7 & 12 & 12 & 91 \\
\hline$\%$ of $n$ & $68 \%$ & $54 \%$ & $8 \%$ & $17 \%$ & $24 \%$ & $29 \%$ & \\
\hline
\end{tabular}

DEX: dexamethasone group.

NON-DEX: non-dexamethasone group.

DEX $\mathrm{n}=50$.

NON-DEX $\mathrm{n}=41$.

Data is missing from two patients (1 from DEX group and 1 from NON-DEX group).

pneumonia $(n=6)$, local infection in gastrostoma $(n=1)$, thrombophlebitis $(n=1)$, surgical wound infection in the neck not needing surgical operation $(n=4)$, and infection of unknown origin $(n=5)$. There was no significant difference in the infection rate between the study group and control group (Table 4). Duration of intravenous antibiotic treatment ranged from three days to 30 days, and the median length was eight days in the DEX group and seven days in the NON-DEX group. A subgroup analysis was performed and there were not more minor or major complications or infections in the diabetic group that got dexamethasone than in the NON-DEX group.

In multivariate analysis, significant associations with recovery time were found for alcohol use and each of the major complications, but the associations were similar for both study groups. According to multivariate logistic regression, not having a major complication (OR 16.1, 95\% CI 2.5-107.9, p=0.004) and higher BMI (OR $1.1,95 \%$ CI $1.0-1.3, \mathrm{p}=0.042$ ) were significantly associ- ated with shorter/better recovery. Those with major use of alcohol (OR $0.07,95 \% \mathrm{CI} 0.01-0.5, \mathrm{p}=0.011$ ) had a significantly lower probability of having a shorter and better recovery than those who did not use any alcohol; the same was true for those with ASA 3 (OR $0.03,95 \% \mathrm{CI} 0.001-0.9, \mathrm{p}=0.046$ ) or ASA 4 (OR $0.01,95 \% \mathrm{CI}$ $0.001-0.2, \mathrm{p}=0.010$ ) compared with those with ASA 1 . No difference was present between the groups in amount of nausea.

We used a two-sided Fisher's exact test with a significance level of 0.05 for retrospective power analysis for a test of the two independent proportions, i.e., occurrence of tracheostomy and perioperative use of dexamethasone. With the slightly unbalanced design ( $n=21$ in the dexamethasone group and $n=26$ in the control group) and the rates observed (41\%/62\%), we estimated a power of 0.81 , which reaches the commonly used standard $(0.80 \leqslant 1-\beta \leqslant 0.95)$. Length of tracheostomy was three days shorter (23\%) in the DEX group, but the difference was not significant. We used a two-sample Satterthwaite $t$-test assuming unequal 
variances with a significance level of 0.05 for retrospective power analysis for a test of the two independent means, i.e., duration of tracheostomy (days) and perioperative use of dexamethasone. With the slightly unbalanced design $(n=20$ in the dexamethasone group and $n=24$ in the control group) and the means observed (8.3 with SD 5.0/11.2 with SD 8.7), we estimated a power of 0.31 , which is clearly below the commonly used standard $(0.80 \leqslant 1-\beta \leqslant 0.95)$.

\section{Discussion}

We performed a prospective double-blind controlled study in head and neck cancer patients with microvascular reconstruction and randomized patients to either receive dexamethasone or not. To our knowledge, this is the first study focusing on the use of GCs in major microvascular reconstruction patients. The patients stayed as long in the intensive care unit and ward, the duration of intubation was the same, and no difference was present in the delay to start of adjuvant radiotherapy between patients receiving dexamethasone and those who did not. There was a trend for a shorter duration of tracheostomy in the DEX group and a power analysis confirmed that the study size was sufficient to conclude that this difference was not statistically significant. We did not find any benefit in the use of dexamethasone with regard to our primary outcome measures.

A systematic review of the literature and meta-analysis by Dan et al. showed that the administration of GCs decreases edema and pain significantly in oral surgery [11]. Pedersen [27], Skjelbred [28], and Milles [29] found a significant decrease in edema but not in pain with low-dose GCs. Neupert et al. reported a significant decrease in pain, but not in edema [30]. We expected the amount of postoperative neck edema, which was measured seven days postoperatively, to be significantly less in the DEX group, but no difference emerged between the groups. In addition, we observed no difference in the amount of nausea between those receiving and not receiving dexamethasone. We therefore conclude that GCs provided no beneficial effects in our head and neck cancer patients undergoing microvascular reconstruction.

Major complications occurred more frequently in patients administered GCs than in the control group. In addition, all infections that needed surgical interventions occurred in patients receiving dexamethasone. Dexamethasone is often given to reduce the risk of postoperative nausea and vomiting, but steroid-induced immunosuppression can increase infection risk by impairing innate immune responses and healing. Previous evidence has also shown contradictory results regarding the influence of perioperative GCs on postoperative complications and infections [23,31,32]. Percival et al. [22] concluded that intraoperative administration of dexamethasone for anti-emetic purposes may confer an increased risk of postoperative infection. The operations in their analysis included orthopedic, thoracic, neurosurgical, otolaryngologic, vascular, plastic, breast, urology, colonic, and gastroenterological procedures, but the amounts of dexamethasone were relatively small. By contrast, the meta-analysis by Dan et al. [11] showed that giving GCs during oral surgery did not significantly increase the risk of infection. In our study, infections treated with antibiotics were similar between the groups, but surgical infections needing secondary surgery within three weeks and classified as major complications occurred more often in the DEX group; this difference was significant.

The randomization in this study succeeded because there were only minor differences in the demographic and perioperative data between the two groups. The only intergroup differences were more heavy alcohol users in the NON-DEX group and more tracheostomized patients in the DEX group. However, four patients in the DEX group and one patient in the NON-DEX group needed to be tracheostomized postoperatively. There was a trend for more diabetics in the DEX group, however in a subgroup analysis diabetics in the DEX group did not have more complications than the NON-DEX group. Since the main finding of this study is the higher number of major complications in the DEX group, we conclude that these differences in patient groups did not confound the result. The patient population with head and neck neoplasms is largely an elderly population, and these patients often have significant histories of smoking and alcohol consumption. These patients are also more likely to have comorbidities. Various predictors of complications have been proposed and investigated widely such as age, histories of smoking and alcohol consumption, comorbidities, preoperative radiation, and chemoradiotherapy. Le Nobel et al. [33] retrospectively analyzed perioperative complications following 304 free flap reconstructions in the head and neck and investigated potential predictors of these complications. They suggested that head and neck free flap reconstruction can be done safely and effectively despite advanced age of the patient and high frequency of comorbid conditions. In our material, multivariate analysis revealed significant associations with recovery time for alcohol use and major complications, but the associations were similar for both study groups. Age and comorbidities did not increase complication risk for free flap patients.

We conclude that in this prospective randomized double-blind study of head and neck cancer patients with microvascular reconstruction the use of dexamethasone did not benefit the patients. Patients receiving dexamethasone did not have shorter visits in the intensive care unit or ward, nor did they show decreased tracheostomy or intubation times. There was no difference in the swelling of the neck between the groups. However, more major complications, especially infections, occurred in patients receiving dexamethasone. Our findings thus do not support the use of periand postoperative dexamethasone in head and neck cancer patients with microvascular reconstruction.

This research did not receive any specific grant from funding agencies in the public, commercial, or not-for-profit sectors.

\section{Conflict of interest}

The authors declare no conflicts of interest.

\section{References}

[1] Panje WR, Bardach J, Krause CJ. Reconstruction of the oral cavity with a free flap. Plast Reconstr Surg 1976;58(4):415-8.

[2] Harashina T, Fujino T, Aoyagi F. Reconstruction of the oral cavity with a free flap. Plast Reconstr Surg 1976;58(4):412-4.

[3] Brown JS, Magennis P, Rogers SN, Cawood JI, Howell R, Vaughan ED. Trends in head and neck microvascular reconstructive surgery in Liverpool (1992-2001). Br J Oral Maxillofac Surg 2006;44(5):364-70.

[4] Urken ML, Weinberg H, Buchbinder D, Moscoso JF, Lawson W, Catalano PJ, et al. Microvascular free flaps in head and neck reconstruction. Report of 200 cases and review of complications. Arch Otolaryngol Head Neck Surg 1994;120 (6):633-40.

[5] Hurvitz KA, Kobayashi M, Evans GR. Current options in head and neck reconstruction. Plast Reconstr Surg 2006;118(5):122e-33e.

[6] Chinn SB, Myers JN. Oral cavity carcinoma: current management, controversies, and future directions. J Clin Oncol 2015;33(29):3269-76.

[7] Scully C, Bagan JV. Recent advances in oral oncology. Oral Oncol 2007;43 (2):107-15.

[8] Neligan PC, Gullane PJ, Gilbert RW. Functional reconstruction of the oral cavity. World J Surg 2003;27(7):856-62.

[9] De Oliveira GS, Jr Almeida MD, Benzon HT, McCarthy RJ. Perioperative single dose systemic dexamethasone for postoperative pain: a meta-analysis of randomized controlled trials. Anesthesiology 2011;115(3):575-88.

[10] Diakos EA, Gallos ID, El-Shunnar S, Clarke M, Kazi R, Mehanna H. Dexamethasone reduces pain, vomiting and overall complications following tonsillectomy in adults: a systematic review and meta-analysis of randomised controlled trials. Clin Otolaryngol 2011;36(6):531-42. 
[11] Dan AE, Thygesen TH, Pinholt EM. Corticosteroid administration in oral and orthognathic surgery: a systematic review of the literature and meta-analysis. J Oral Maxillofac Surg 2010;68(9):2207-20.

[12] Hussain A, Young WB. Steroids and aseptic osteonecrosis (AON) in migraine patients. Headache 2007:47(4):600-4.

[13] Chan MH, Chan PK, Griffith JF, Chan IH, Lit LC, Wong CK, et al. Steroid-induced osteonecrosis in severe acute respiratory syndrome: a retrospective analysis of biochemical markers of bone metabolism and corticosteroid therapy. Pathology 2006;38(3):229-35.

[14] Wong GK, Poon WS, Chiu KH. Steroid-induced avascular necrosis of the hip in neurosurgical patients: epidemiological study. ANZ J Surg 2005;75(6):409-10.

[15] Natkunarajah J, Goolamali S, Craythorne E, Benton E, Smith C, Morris-Jones R, et al. Ten cases of drug reaction with eosinophilia and systemic symptoms (DRESS) treated with pulsed intravenous methylprednisolone. Eur J Dermatol 2011;21(3):385-91.

[16] Ularntinon S, Tzuang D, Dahl G, Shaw RJ. Concurrent treatment of steroidrelated mood and psychotic symptoms with risperidone. Pediatrics 2010;125 (5):e1241-5.

[17] Fleming PS, Flood TR. Steroid-induced psychosis complicating orthognathic surgery: a case report. Br Dent J 2005;199(10):647-8.

[18] Flood TR, McManners J, el-Attar A, Moos KF. Randomized prospective study of the influence of steroids on postoperative eye-opening after exploration of the orbital floor. Br J Oral Maxillofac Surg 1999;37(4):312-5.

[19] Galen DM, Beck M, Buchbinder D. Steroid psychosis after orthognathic surgery: a case report. J Oral Maxillofac Surg 1997;55(3):294-7.

[20] O'Neil EA, Chwals WJ, O'Shea MD, Turner CS. Dexamethasone treatment during ventilator dependency: possible life threatening gastrointestinal complications. Arch Dis Child 1992;67(1 Spec No):10-1.

[21] Olsen M, Christensen S, Riis A, Thomsen RW. Preadmission use of systemic glucocorticoids and 30-day mortality following bleeding peptic ulcer: a population-based cohort study. Am J Ther 2010;17(1):23-9.

[22] Percival VG, Riddell J, Corcoran TB. Single dose dexamethasone for postoperative nausea and vomiting-a matched case-control study of postoperative infection risk. Anaesth Intensive Care 2010;38(4):661-6.
[23] Snall J, Kormi E, Koivusalo AM, Lindqvist C, Suominen AL, Tornwall J, et al. Effects of perioperatively administered dexamethasone on surgical wound healing in patients undergoing surgery for zygomatic fracture: a prospective study. Oral Surg Oral Med Oral Pathol Oral Radiol 2014;117(6):685-9.

24] Mastropietro CW, Barrett R, Davalos MC, Zidan M, Valentine KM, Delius RE, et al. Cumulative corticosteroid exposure and infection risk after complex pediatric cardiac surgery. Ann Thorac Surg 2013;95(6):2133-9.

[25] Dindo D, Demartines N, Clavien PA. Classification of surgical complications: a new proposal with evaluation in a cohort of 6336 patients and results of a survey. Ann Surg 2004;240(2):205-13.

[26] Clavien PA, Barkun J, de Oliveira ML, Vauthey JN, Dindo D, Schulick RD, et al. The Clavien-Dindo classification of surgical complications: five-year experience. Ann Surg 2009;250(2):187-96.

[27] Pedersen A. Decadronphosphate in the relief of complaints after third molar surgery. A double-blind, controlled trial with bilateral oral surgery. Int J Oral Surg 1985;14(3):235-40.

[28] Skjelbred P, Lokken P. Reduction of pain and swelling by a corticosteroid injected 3 hours after surgery. Eur J Clin Pharmacol 1982;23(2):141-6.

[29] Milles M. Desjardins P. Reduction of postoperative facial swelling by low-dose methylprednisolone: an experimental study. J Oral Maxillofac Surg 1993;51 (9):987-91.

[30] Neupert 3rd EA, Lee JW, Philput CB, Gordon JR. Evaluation of dexamethasone for reduction of postsurgical sequelae of third molar removal. J Oral Maxillofac Surg 1992;50(11):1177-82. discussion 1182-3.

[31] Waldron NH, Jones CA, Gan TJ, Allen TK, Habib AS. Impact of perioperative dexamethasone on postoperative analgesia and side-effects: systematic review and meta-analysis. Br J Anaesth 2013;110(2):191-200.

[32] Richardson AB, Bala A, Wellman SS, Attarian DE, Bolognesi MP, Grant SA. Perioperative dexamethasone administration does not increase the incidence of postoperative infection in total hip and knee arthroplasty: a retrospective analysis. J Arthroplasty 2016;31(8):1784-7.

[33] le Nobel GJ, Higgins KM, Enepekides DJ. Predictors of complications of free flap reconstruction in head and neck surgery: analysis of 304 free flap reconstruction procedures. Laryngoscope 2012;122(5):1014-9. 